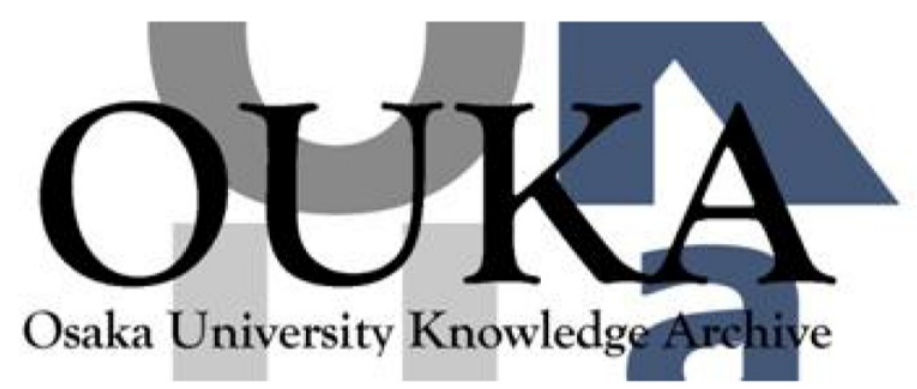

\begin{tabular}{|c|c|}
\hline Title & $\begin{array}{l}\text { Experimental evidence of nonthermal } \\
\text { acceleration of relativistic electrons by an } \\
\text { intensive laser pulse }\end{array}$ \\
\hline Author (s) & Kuramitsu, Y.; Nakani i, N.; Kondo, K. et al. \\
\hline Citation & Physical Review E. 83(2) p.026401 \\
\hline Issue Date & $2011-02$ \\
\hline oaire:version & VoR \\
\hline URL & https://hdl. handle. net/11094/3450 \\
\hline rights & 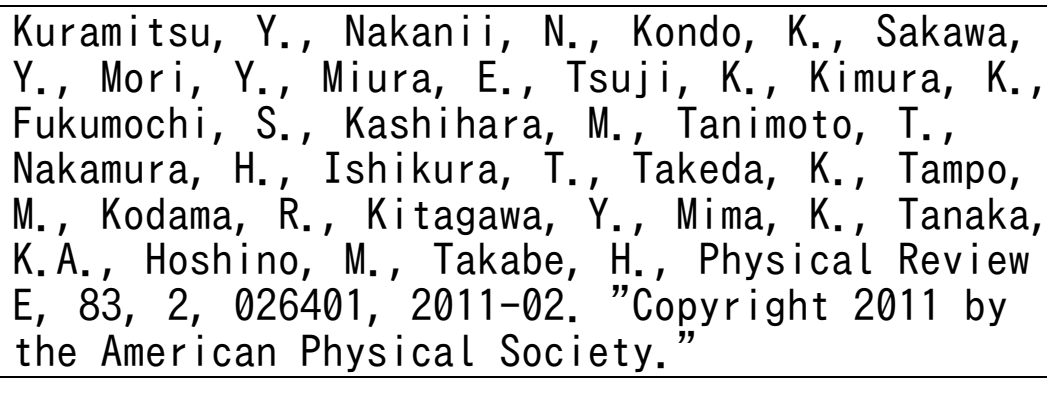 \\
\hline Note & \\
\hline
\end{tabular}

Osaka University Knowledge Archive : OUKA

https://ir. Library. osaka-u. ac. jp/

0saka University 


\title{
Experimental evidence of nonthermal acceleration of relativistic electrons by an intensive laser pulse
}

\author{
Y. Kuramitsu, ${ }^{1, *}$ N. Nakanii, ${ }^{1,2}$ K. Kondo, ${ }^{1,2}$ Y. Sakawa, ${ }^{1}$ Y. Mori, ${ }^{1,3}$ E. Miura, ${ }^{4}$ K. Tsuji, ${ }^{1,2}$ K. Kimura, ${ }^{1,2}$ S. Fukumochi, ${ }^{1,2}$ \\ M. Kashihara, ${ }^{1,2}$ T. Tanimoto, ${ }^{1,2}$ H. Nakamura, ${ }^{1,2}$ T. Ishikura, ${ }^{1,2}$ K. Takeda, ${ }^{1}$ M. Tampo, ${ }^{1}$ R. Kodama, ${ }^{1,2}$ Y. Kitagawa, ${ }^{3}$ \\ K. Mima, ${ }^{1,2}$ K. A. Tanaka, ${ }^{1,2}$ M. Hoshino, ${ }^{5}$ and H. Takabe ${ }^{1}$ \\ ${ }^{1}$ Institute of Laser Engineering, Osaka University, 2-6 Yamadaoka, Suita, Osaka 565-0871, Japan \\ ${ }^{2}$ Graduate School of Engineering, Osaka University, 2-1 Yamadaoka, Suita, Osaka 565-0871, Japan \\ ${ }^{3}$ The Graduate School for the Creation of New Photonics Industries, Hamamatsu, Shizuoka 432-1202, Japan \\ ${ }^{4}$ National Institute of Advanced Industrial Science and Technology, Tsukuba, Ibaraki 305-8568, Japan \\ ${ }^{5}$ Department of Earth and Planetary Science, The University of Tokyo, 7-3-1 Hongo, Bunkyo, Tokyo 113-0033, Japan
}

(Received 8 May 2009; revised manuscript received 15 November 2010; published 4 February 2011)

\begin{abstract}
Nonthermal acceleration of relativistic electrons is investigated with an intensive laser pulse. An energy distribution function of energetic particles in the universe or cosmic rays is well represented by a power-law spectrum, therefore, nonthermal acceleration is essential to understand the origin of cosmic rays. A possible candidate for the origin of cosmic rays is wakefield acceleration at relativistic astrophysical perpendicular shocks. The wakefield is considered to be excited by large-amplitude precursor light waves in the upstream of the shocks. Substituting an intensive laser pulse for the large amplitude light waves, we performed a model experiment of the shock environments in a laboratory plasma. An intensive laser pulse was propagated in a plasma tube created by imploding a hollow polystyrene cylinder, as the large amplitude light waves propagated in the upstream plasma at an astrophysical shock. Nonthermal electrons were generated, and the energy distribution functions of the electrons have a power-law component with an index of $\sim 2$. We described the detailed procedures to obtain the nonthermal components from data obtained by an electron spectrometer.
\end{abstract}

DOI: 10.1103/PhysRevE.83.026401

PACS number(s): $52.72 .+\mathrm{v}, 52.27 . \mathrm{Ny}, 52.38 . \mathrm{Kd}, 98.70 . \mathrm{Sa}$

\section{INTRODUCTION}

Ever since the discovery of cosmic rays, a number of scientists in space, astro-, and plasma physics have intensively and extensively investigated the origins of the cosmic rays for more than a century. The diffusive shock acceleration or the first-order Fermi acceleration naturally reproduces a power-law spectrum of accelerated particles with an index of 2 and is widely accepted as a standard acceleration mechanism of cosmic rays within our galaxy [1-3]. However, the origin of extragalactic cosmic rays has been an open unsolved problem. High energetic phenomena in the universe, such as active galactic nuclei (AGN), $\gamma$-ray bursts, and pulsars are considered to be energy sources for the extragalactic cosmic rays, and a possible candidate of the cosmic ray acceleration is the wakefield acceleration in such astrophysical environments [4]. In the universe, large-amplitude Alfvén waves can excite a wakefield, then the wakefield can accelerate charged particles efficiently [5]. In the upstream of relativistic perpendicular shock waves, efficient particle acceleration is possible due to wakefields induced by large-amplitude precursor electromagnetic (light) waves [6]. So far, however, there is no way to experimentally or observationally confirm these theories or analytic models on cosmic ray acceleration since those astrophysical shocks are never accessible by direct measurements of field and plasma quantities; those are crucial for understanding the transport properties of the cosmic rays [7]. Laboratory experiments are alternative ways to investigate unaccessible astrophysical phenomena in the universe [8-11].

*kuramitsu-y@ile.osaka-u.ac.jp
The wakefield acceleration was first proposed in Ref. [12], and laser-based particle acceleration has been investigated extensively, such as nearly monoenergetic electron acceleration [13-17] and direct electron acceleration by tightly focused laser pulses [18-21]. In these processes, the laser and plasma conditions have to be well controlled to have a comparable or smaller spot size than the plasma wavelength. On the other hand, in a relativistic perpendicular shock with the upstream bulk Lorentz factor $\gamma_{1} \gg 1$, large-amplitude electromagnetic (light) waves are known to be excited due to the synchrotron maser instability. These waves propagate toward upstream of the shock as precursor waves and excite wakefield due to the ponderomotive force of the light waves. Hoshino [6] reported the efficient acceleration of particles by the wakefield in the shock environment using a one-dimensional particle-in-cell (PIC) simulation. Kuramitsu et al. [22] extended the previous work using two-dimensional PIC simulations, by focusing on the wakefield acceleration of electrons with a large-amplitude light (laser) pulse. In contrast to the current laser plasma accelerators, it is expected that the light waves excited in the upstream of the shock have larger spatial scale than the electron inertial scale since the shock scales are much larger than the electron scale. Thus, on modeling an astrophysical shock upstream, an intensive light wave (laser) substituted for the large-amplitude light waves has to have larger spatial and temporal scales than the electron plasma wave. When a laser pulse is longer than a plasma wavelength, the self-modulation of the light pulse is known to take place, and the modulated light wave can self-consistently excite the wakefield. When the laser spot size is larger than the plasma wavelength, the filamentation of the laser pulse is known to occur, and then, each filamented part of the light can excite the wakefield. In such a field, the electrons are nonthermally accelerated by 
the wakefield, resulting in a power-law energy spectra with an index of $\sim 2$, independent of the laser and the plasma conditions as long as the laser intensity is relativistic [22].

In this paper, we report the experimental evidence of nonthermal acceleration for electrons using an intensive laser pulse. The energetic electrons are produced by the Gekko petawatt (PW) laser propagating in a plasma tube generated with six beams of the Gekko XII (GXII) laser system. The density of the plasma tube was controlled by varying the total energy of the GXII laser. We describe how to evaluate the energy spectra of accelerated electrons in detail. The energy distribution functions of the electrons have a power-law component with an index of $\sim 2$, independent of the implosion energy or the plasma density as predicted by the numerical simulations [22].

\section{EXPERIMENTS}

The experiment was performed using the Gekko PW laser system at the Institute of Laser Engineering at Osaka University [23]. A plasma tube was preformed by imploding a hollow polystyrene plastic cylinder (the length of $3 \mathrm{~mm}$, the diameter of $700 \mu \mathrm{m}$, and the wall thickness of $14 \mu \mathrm{m}$ ) by six beams of the GXII laser (the wavelength of $532 \mathrm{~nm}$ and the pulse length of $1 \mathrm{~ns}$ ) [24]. The plasma tube, which consists of a central low-density region surrounded by an outer high-density region, is formed during the implosion. The pump laser beam from the PW laser system was injected $3.1 \mathrm{~ns}$ after the irradiation of the implosion beams, and this timing was fixed for all shots. The focal spot size of the pump laser measured by an X-ray pinhole camera was $65 \mu \mathrm{m}$. The PW laser system is a chirped-pulse-amplification Nd:glass laser system having a central wavelength of $1.053 \mu \mathrm{m}$. In the experiment, the pump beam had a pulse energy of $100 \mathrm{~J}$ and a full width at half maximum of $700 \mathrm{fs}$ at the target. The pump laser beam from the PW laser system was injected into the plasma tubes with different densities in their central regions as $9 \times 10^{18}, 2 \times 10^{19}$, and $3.7 \times 10^{19} \mathrm{~cm}^{-3}$, by varying the total energy of the implosion beams, $E_{\text {imp }}=1.9,2$, and $2.3 \mathrm{~kJ}$, respectively [24]. The energy spectrum of electrons accelerated forward along the propagation axis of the pump beam was measured using an electron spectrometer (ESM), which uses a 1-T permanent dipole magnet and a FUJIFILM
BAS-SR2025 imaging plate (IP) as a detector. Figure 1 schematically shows the ESM. To measure $\sim 1-\mathrm{GeV}$ electrons, a 470-mm-long magnet was used. A 100-mm-long stainless steel block with an 8-mm-diameter tube was placed at the entrance of the ESM to collimate the electrons. The collection solid angle was $2.8 \times 10^{-5}$ sr. A $100-\mathrm{mm}$-thick lead shield was placed between the interaction chamber and the detector to reduce the background signal. The IP was covered with a $12-\mu \mathrm{m}$-thick aluminum film to prevent it from being exposed to visible and/or UV radiation. The IPs were placed both on the electron side and on the ion side in the ESM. The IPs were scanned using a FUJIFILM BAS-1800 scanner having a 200- $\mu \mathrm{m}$ spatial resolution. The absolute calibration of the IP was performed using a linac at Osaka University for energies below $100 \mathrm{MeV}$ [25] and with a linac at SPring-8 for energies in the range of $100 \mathrm{MeV}$ to $1 \mathrm{GeV}$ [26].

\section{RESULTS}

Figure 2(a) shows the intensities of photostimulated luminescence (PSL) $I_{\mathrm{PSL}}$, which is proportional to $F \Delta E$ ( $F$, energy distribution function, $\Delta E$, energy bin), detected on the electron side (solid line) and on the ion side (dotted line). When a high-energy component of energy distribution function is discussed, a logarithmic plot to the base 10 must be used. In Fig. 2(a), the electron-side signals almost overlap with the ion-side signals for the electron energy $>100 \mathrm{MeV}$, but the electron-side signals are slightly more than the ion-side signals. The ion-side signals are considered to come from the $\mathrm{X}$ and $\gamma$ rays from the laser-plasma interactions at the chamber center, and from that, the accelerated electrons hit the collimator at the entrance of the ESM. The former $\gamma$ rays hit the far-side wall of the ESM (near the window in Fig. 1) and then produce the secondary $\gamma$ ray detected in the farthest side of ESM in Fig. 2. The latter $\gamma$ rays go through the collimator and can be detected on the IPs. The collimator surface with the diameter of $40.6 \mathrm{~mm}$ can be an area source for the $\gamma$ rays, thus, an ideal point source for the $\gamma$ rays is considered to be at $\sim 40 \mathrm{~mm}$ away from the collimator surface. Figure 2(b) shows the ion-side signals against the distance $r$ form a nominal point source $(40 \mathrm{~mm}$ to the chamber center from the collimator surface). The intensity of PSL shows $r^{-2}$ decay, ensuring that the ion-side signals mostly come from the $\gamma$ rays created

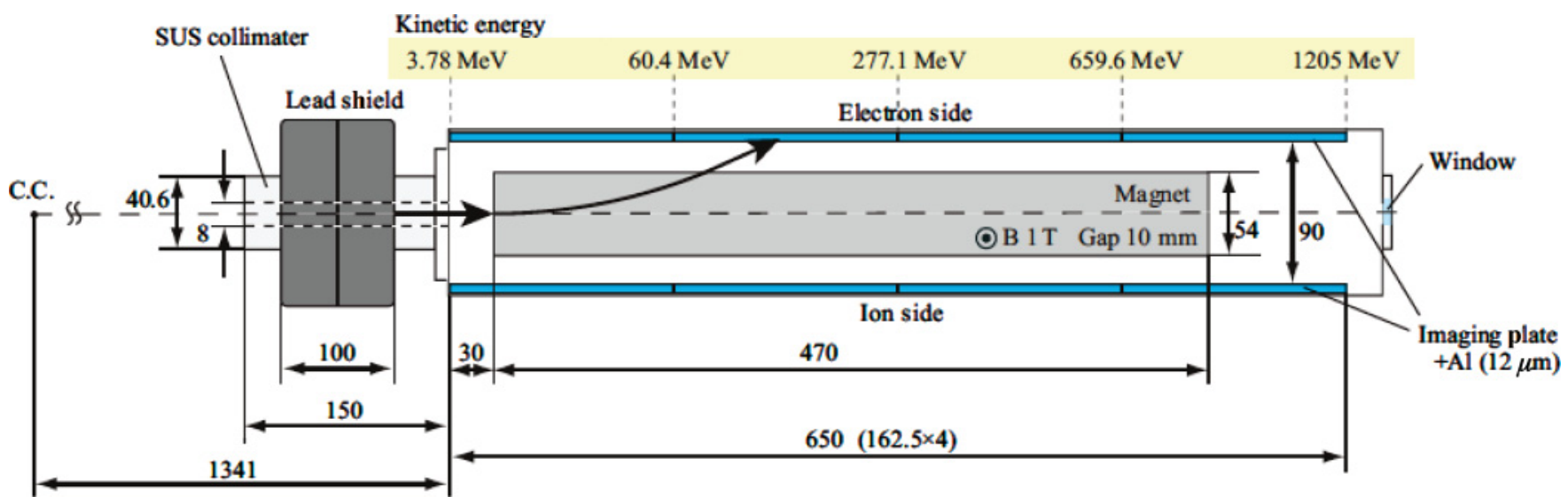

FIG. 1. (Color online) Schematic of the ESM. 

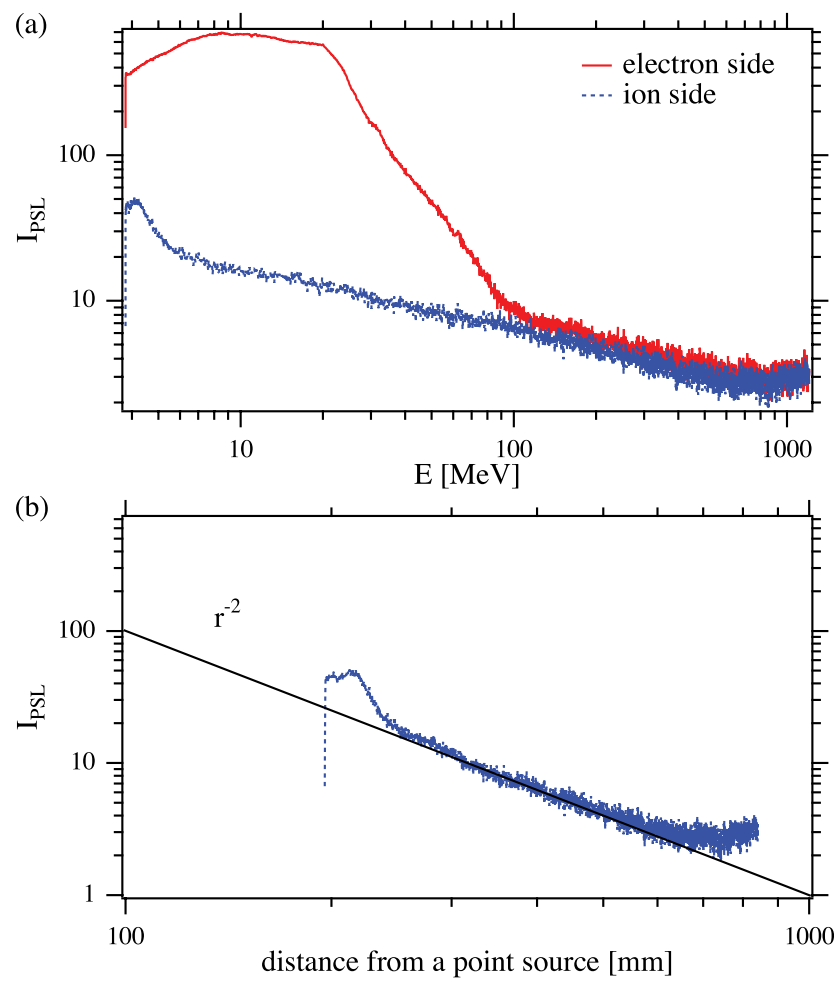

FIG. 2. (Color online) (a) Intensities of PSL of the electron side and the ion side against electron energy in MeV. (b) Intensity of PSL of the ion side against the distance from a point source of $\gamma$ rays.

by energetic electrons at the surface of the collimator. The electron-side signals consist of the accelerated electrons and the $\gamma$ rays from the entrance and the end wall of the ESM. The high-energy part of the distribution functions of the accelerated electrons is expected to have a power-law component from the numerical simulations [22]. Thus, since the intensity of PSL is recorded on a linear scale on the IPs, both the signals from the energetic electrons and the $\gamma$ rays decrease as the power of the distance and of the electron energy; the signals on both sides become less and less as the electron energy increases. The raw data on IPs in Fig. 2(a) are poor for discussing the high-energy components in ESM; a fluctuated spectrum is always less reliable.

In order to increase the statistics in the high-energy region, one can reevaluate the raw data by using logarithmic bins. Figure 3(a) shows the reevaluated data with logarithmic bins for the shot with $E_{\mathrm{imp}}=1.9 \mathrm{~kJ}$. We use 100 grids in $1 \leqslant \gamma \leqslant 3000$ with a constant $\Delta \log _{10} \gamma$, where $\gamma$ is the electron Lorentz factor. This increases the statistics in the high-energy region and also removes the fluctuations in the data. Note that none of the signals show $\gamma^{-2}$ dependence in Fig. 3(a). Now, we can clearly see the symmetry between the electron-side data and the ion-side data; the electron-side signals asymptotically approach the ion-side signals and merge with them at $\gamma \sim 2000$. Figures 3(b)-3(d) show the highenergy part of the data for three different shots. All three cases show the same characteristics; two signals form the electron, and ion sides asymptotically merge together. If they are not symmetric due to, e.g., a small-angle scattering at the collimator, the electron-side signals must be more than the

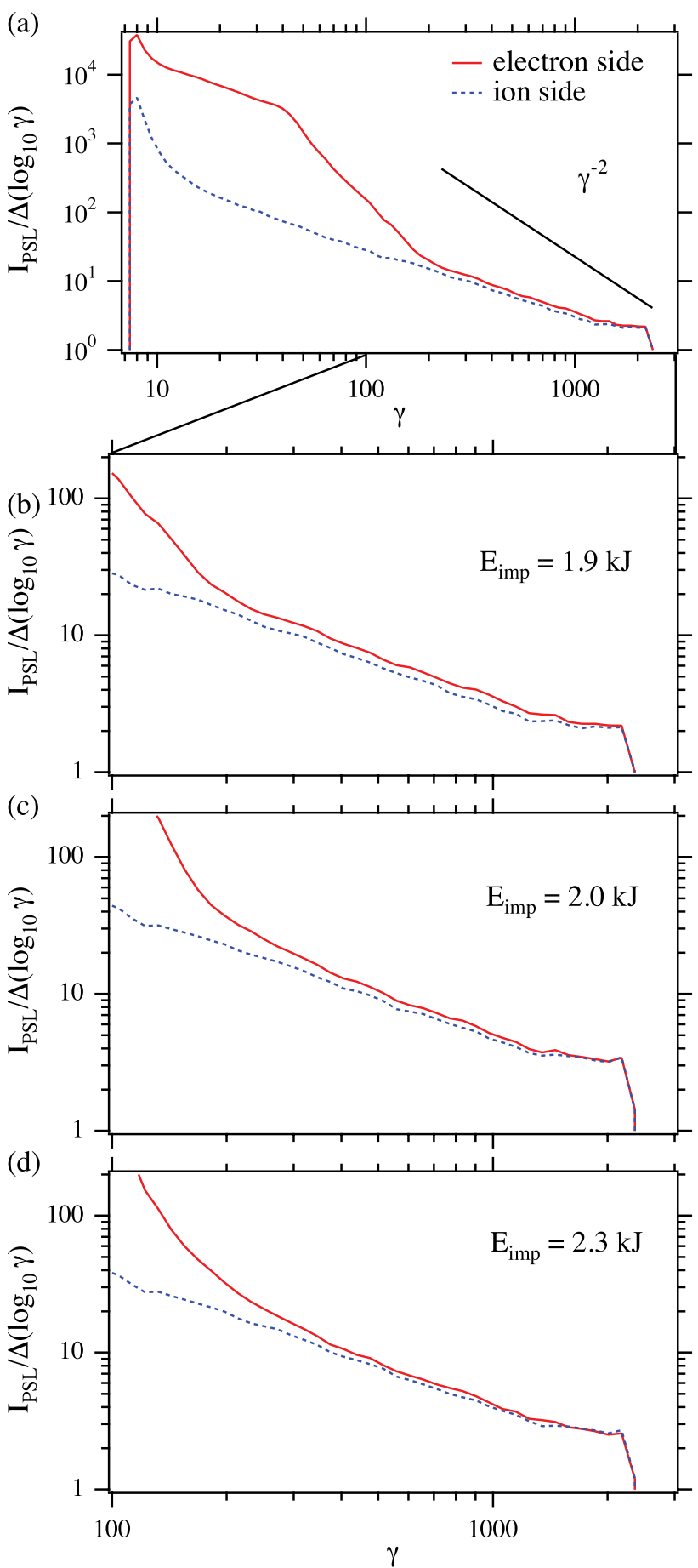

FIG. 3. (Color online) (a) Reevaluation of ESM data using logarithmic bins. $E_{\text {imp }}=1.9 \mathrm{~kJ}$. (b) Expanded plot of high-energy region of (a). (c) Same as (b) except for $E_{\mathrm{imp}}=2.0 \mathrm{~kJ}$. (d) Same as (b) except for $E_{\text {imp }}=2.3 \mathrm{~kJ}$.

ion-side ones in the entire region of the spectra. The higher implosion energy, i.e., the higher plasma density is the lower merging energy: $\gamma \sim 2000,1500$, and 1000 for $E_{\mathrm{imp}}=1.9,2$, and $2.3 \mathrm{~kJ}$, respectively.

Figure 4(a) shows the accelerated electron spectra obtained by subtracting the ion-side signals from the electron-side signals. The dashed, dotted, and solid lines represent $E_{\mathrm{imp}}=$ $1.9,2.0$, and $2.3 \mathrm{~kJ}$, respectively. All three spectra for the 

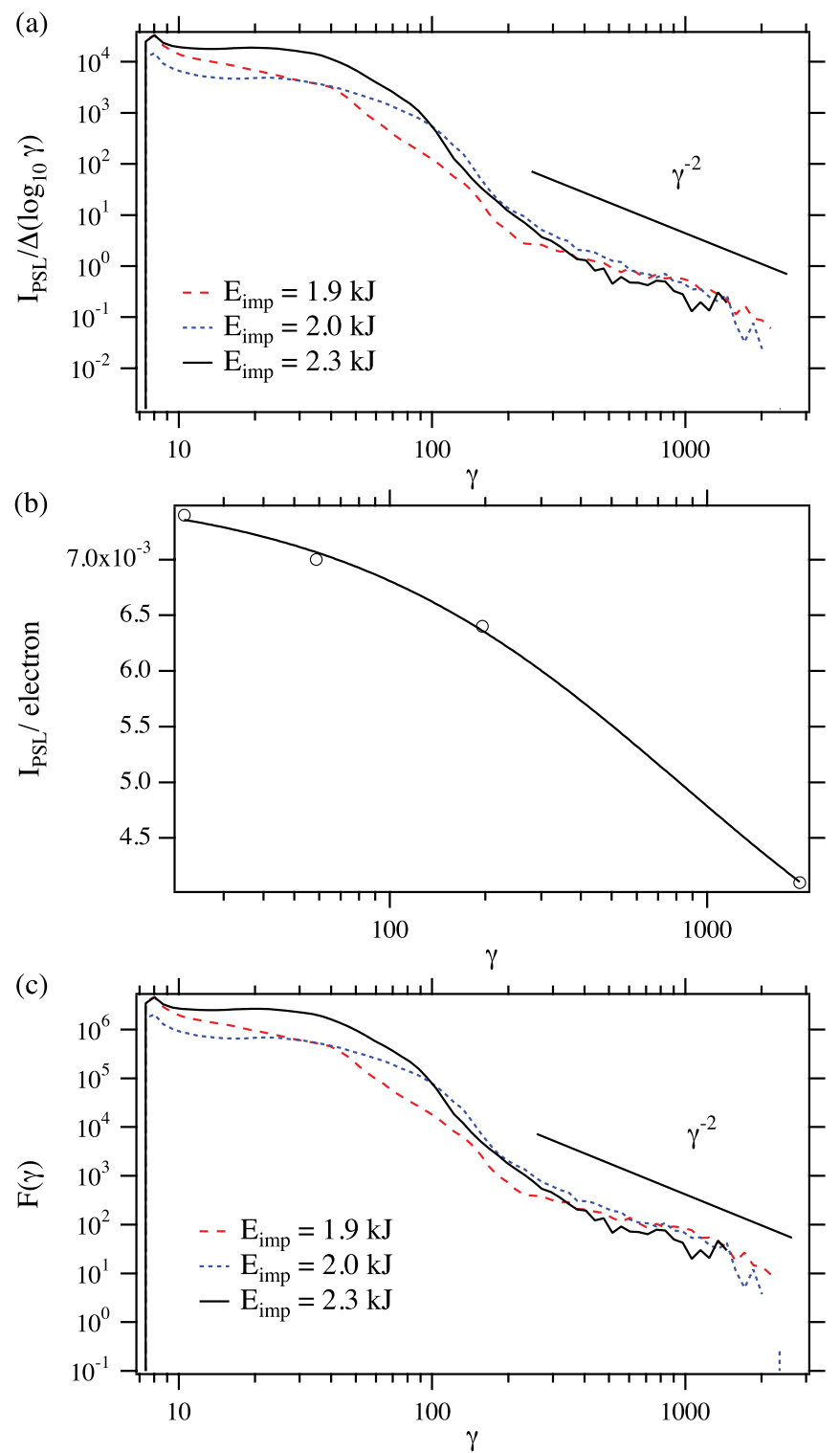

FIG. 4. (Color online) (a) ESM signals after subtracting the ionside values from the electron-side values. (b) IP sensitivity obtained from absolute calibrations (open circles) and the fitted line. (c) Energy distribution functions of electrons.

accelerated electrons show $\gamma^{-2}$ dependence in the high-energy part. The higher implosion energy is the lower maximum energy in Fig. 4(a) as expected from Figs. 3(b)-3(d). In Fig. 4(b), the open circles show the IP sensitivities for $\gamma \leqslant 200$ in Ref. [25] and for $\gamma \sim 2000$ in Ref. [26], and the solid line is the fitted curve. Dividing the spectra in Fig. 4(a) by the fitted function of the absolute calibrations in Fig. 4(b), we obtain the energy distribution function of the accelerated electrons $F(\gamma)$ in Fig. 4(c). Since the sensitivities in Fig. 4(b) do not depend on the energy much (less than a factor of 2), Figs. 4(a) and 4(b) do not change much in terms of the index of power law; Fig. 4(c) also shows $\gamma^{-2}$ dependence.

The distribution functions consist of two parts; one is the thermal part, and the other is the nonthermal part, which is represented as a power law. The thermal part of the spectra was discussed in Ref. [24]; the electron spectra for $E_{\text {imp }}=$
$2.3 \mathrm{~kJ}$ have a peak structure, which is not clear in Fig. 4(c) with the logarithmic plot, around $\gamma \sim 20$ in the continuous spectrum. The peak formation is explained by the trapping of the electrons by the wakefield excited by the pump laser and is reproduced by a two-dimensional particle simulation. While the thermal parts in the distribution functions show different features depending on the implosion energies, the nonthermal parts show a common feature, a power law with an index of $\sim 2$ independent of the plasma density.

\section{DISCUSSION}

An astrophysical shock scale can be regarded as infinite, and the time scale is also considered to be infinite when compared with the electron scale length. In such a system, a light wave propagating in the upstream plasma of the relativistic shock is well described by a plane wave with the dispersion relation with $\omega_{L}^{2}-k_{L}^{2} c^{2}=\omega_{p}^{2} / \gamma_{0}$, where $\omega_{L}$ is the angular frequency of the light wave, $k_{L}$ is the wave number of the light wave, $c$ is the speed of light in vacuum, $\omega_{p}$ is the nonrelativistic plasma frequency, $\gamma_{0} \equiv\left(1+a_{0}^{2} / 2\right)^{1 / 2}$ is the Lorentz factor of the quivering electrons, and $a_{0}$ is the normalized light amplitude. When the light amplitude is large, the strong ponderomotive force of the light can excite a coherent wakefield, propagating at the same velocity as the light group velocity, $v_{\phi}=\omega_{p} / k_{p}=c \eta$, where $k_{p}$ is the wave number of the plasma wave, and $\eta \equiv\left[1-\omega_{p}^{2} /\left(\omega_{L}^{2} \gamma_{0}\right)\right]^{1 / 2}$. In the wave-breaking limit $e E \sim m c \omega_{p}$, where $e$ is the electron charge, $E$ is the amplitude of the wake electric field, and $m$ is the electron mass; the maximum energy of an electron in the wake frame is written as $\gamma_{\max }^{\prime}=2 \gamma_{\phi} \eta+1$, where $\gamma_{\phi}=$ $\left(1-v_{\phi}^{2} / c^{2}\right)^{-1 / 2}=\omega_{L} \gamma_{0}^{1 / 2} / \omega_{p}$, and, in the plasma frame, is written as $\gamma_{\max }=\gamma_{\phi}\left[\gamma_{\max }^{\prime}+\eta\left(\gamma_{\max }^{\prime 2}-1\right)^{1 / 2}\right]$. Therefore, in the presence of a coherent wakefield, the maximum attainable energy in such a field is limited [12]. In our experiment, $a_{0}=$ 1.87 and $\omega_{L} / \omega_{p}=5.21,7.09$, and 10.6 for $E_{\mathrm{imp}}=2.3,2$, and $1.9 \mathrm{~kJ}$; the $\gamma_{\max }$ is expected as 190,348 , and 765 , respectively. These are much smaller than the maximum energies in Fig. 4(c), where the electrons are nonthermally accelerated up to $\gamma \gtrsim 1000$ for all the implosion energies. Although the conventional wakefield acceleration cannot explain the nonthermal acceleration of electrons quantitatively, the density dependence of the acceleration is qualitatively consistent with the experimental result; the lower density is the higher energy.

Chen et al. [4] reported a statistical model for the cosmic ray acceleration due to wakefield acceleration. In their model, they assume a purely stochastic process where the probability transition rate of the energy distribution function of particles depends neither on the particle energy nor on the energy gain by the acceleration process. This gives the energy distribution function with power-law scaling an index of 2 . As they considered a stochastic wakefield, the original model of wakefield acceleration [12] might not be directly applicable to the cosmic ray acceleration, since, in a coherent field, a particle just oscillates in the wake frame without any energization; energy diffusion must be concerned for the cosmic ray acceleration. In astrophysical shocks, the wakefield can be more irregular since the plasma density can be different from place to place in astrophysical scales, and the light wave amplitudes can 
also differ depending on place and time. Thus, the stochastic wakefield or turbulent wakefield is an appropriate description of the field in astrophysical circumstances. In our experiment, an incoherent wakefield is also expected to be excited as seen in the numerical experiments in Refs. [6,22]. Here, we use the term incoherent since our relevant numerical study [22] shows not coherent but not completely irregular wakefield where electrons are accelerated in each bubble located $\sim 2 \pi c / \omega_{p}$ (Fig. 1 in Ref. [22]), i.e., the probability transition strongly depends on the particle velocity. Although it is not completely turbulent, the spectra of the accelerated electrons show powerlaw nature independent of the plasma and laser conditions [22]. In order to understand this, we need further theoretical analysis on the universality of the nonthermal acceleration of electrons.

In a turbulent field, for example, in magnetohydrodynamics turbulence, a particle repeats resonant scattering and diffuses in the momentum space, or the energy diffusion takes place $[27,28]$. In a field where the wave spectrum has a finite width but a certain mode is dominant, a particle resonant with the dominant mode or a trapped particle by the dominant mode is unstable in the phase space when the particle is on or close to the separatrix. The separatrix is unstable to linear perturbations, i.e., the energy diffusion takes place around the separatrix [29]. In a wakefield, particles obtaining the maximum energy discussed previously are on the separatrix in the phase space. This is the reason why the conventional model is qualitatively consistent with the experimental result, but in the experiment, the electron acceleration is not necessarily limited to this model since the energy diffusion is essential in a field not completely coherent and in a turbulent field.

As discussed before, the governing parameters of wakefield acceleration are the normalized light wave amplitude $a_{0}$ and the frequency ratio between plasma and laser $\omega_{p} / \omega_{L}$. In Ref. [6], the light wave amplitude excited at the astrophysical shock is estimated as $a_{0} \sim \gamma_{1}$, and the frequency ratio is estimated as $\omega_{L} / \omega_{p} \simeq 2 \gamma_{1}$ in the relativistic astrophysical shock upstream both from the numerical simulation. While the Lorentz factors of AGN jets are 10-20 [30], in the experiment, $a_{0}=1.87$ and $\omega_{L} / \omega_{p}=5.21,7.09$, and 10.6 for three different implosion energies $E_{\mathrm{imp}}=2.3,2$, and $1.9 \mathrm{~kJ}$; these are applicable to the AGN jets. Moreover, Kuramitsu et al. [22] reported a universality of power-law acceleration with respect to $a_{0}$ and $\omega_{p} / \omega_{L}$; the wakefield acceleration is widely applicable to high-energy astrophysical phenomena.

The limitation of the model experiment is the spatial and temporal scales of the laser pulse; those are considered to be much smaller than the astrophysical shock precursor waves. However, when a laser pulse is much longer in the longitudinal (laser propagation) direction than a plasma wavelength $\sim 2 \pi c / \omega_{p}$, self-modulation of the laser pulse takes place at the plasma wavelength. The modulated light wave can self-consistently excite the plasma wave (wakefield). In the transverse direction, when the laser scale $>2 \pi c / \omega_{p}$, filamentation of the laser pulse occurs, and then each filamented part of the light effectively acts as a light pulse, resulting in excitation of the wakefield. These self-modulations and filamentations can also make the wakefield irregular. Thus, then a laser pulse with the spot size (transverse scale) $\gg 2 \pi c / \omega_{p}$ and the pulse duration (longitudinal scale) $\gg 2 \pi / \omega_{p}$ can be regarded as a part of the light precursor waves in the astrophysical shock waves. In order to apply a laser experiment to cosmic ray acceleration, the laser pulse must have large scales and large amplitude $\left(a_{0}>1\right)$. In our experiment, the laser spot size $(65 \mu \mathrm{m})$ and the pulse duration (700 fs) are larger than the plasma wave scales (5-11 $\mu \mathrm{m}$ and 18-37 fs).

The conditions to obtain power-law spectra and to apply them to astrophysics are the normalized wave amplitude $a_{0}>1$ and larger spatial scale of the light pulse than the plasma wavelength, i.e., focal spot size $>2 \pi c / \omega_{p}$ and pulse duration $>2 \pi / \omega_{p}$. In such conditions, the energy diffusion due to wakefield can naturally occur and universally results in power-law acceleration. In contrast, when a laser pulse is tightly focused $\left(<2 \pi c / \omega_{p}\right)$, then the direct laser acceleration of particles is possible. Such small scale pulses might not naturally exist in the universe. For example, while the spot size in Ref. [21] is 3.2-5.3 $\mu \mathrm{m}$, in our experiment, it was $65 \mu \mathrm{m}$, which is an order of magnitude larger than the one in Ref. [21]. Furthermore, even by such a tightly focused laser pulse, when the laser intensity is moderate $a_{0} \lesssim 13$, which is much larger than in our case, the wakefield acceleration is dominant [21]. Nevertheless, it is not necessary to exclude the possibility of the direct acceleration of particles by laser pulse or light waves, since the most important result is the nonthermal acceleration of particles when a large amplitude light pulse propagates in a plasma, which is considered to be common in the upstream of an astrophysical shock. If the light waves can nonthermally accelerate particles, resulting in a power-law spectrum, it is still interesting in terms of the cosmic ray acceleration.

In our experiment, the large spot size $(65 \mu \mathrm{m})$ observed by the X-ray pinhole camera provided a necessary condition to apply the wakefield acceleration to the astrophysical shocks and also made our experiment different from those experiments where the laser pulses are tightly focused. The transmitted light showed a broader spectrum than the one of the incident light, ensuring that the plasma wave spectrum must also have a certain width rather than a $\delta$ function, such as the spectrum (not shown). These diagnostics provide necessary information to discuss the acceleration mechanism, however, it is not sufficient to determine it. One has to observe electric and magnetic fields that are considered to produce the energy distribution function of the accelerated particles. A coherent wakefield has been directly observed by Matlis et al. [31]. It is very challenging to observe a turbulent or an irregular coherent wakefield, however, it is still possible. On the contrary, in astrophysical shocks, it is impossible to directly measure the local wakefield; laboratory experiments with high-power lasers have a great advantage for investigating the acceleration of cosmic rays.

\section{SUMMARY}

We have presented the experimental evidence of nonthermal acceleration of electrons by the intensive laser pulse. A plasma tube was created by imploding a plastic cylinder with six long laser pulses prior to the short intensive laser pulse. The plasma density was changed by varying the energy of the implosion beams. The short laser pulse with large spatial and temporal scales, compared with the electron plasma scales, propagated in the preformed plasma and accelerated the plasma electrons. The accelerated electrons were detected 
by the electron spectrometer located on the laser propagation axis. As a leading numerical study predicted [22], the energy distribution functions of the accelerated electrons showed power-law spectra in the high-energy parts with an index of $\sim 2$ independent of the plasma density. Although the maximum energies observed in the experiment were much larger than the estimations from the coherent field, the conventional model qualitatively explains the density dependence of the maximum energy, i.e., the lower density of plasma is the higher maximum energy of electrons, implying that the energy diffusion is essential to the nonthermal acceleration of electrons.

\section{ACKNOWLEDGMENTS}

The authors would like to thank T. Hada, S. Matsukiyo, T. N. Kato, and C. Joshi for their helpful comments.
[1] W. I. Axford, E. Leer, and G. Skadron, in International Cosmic Ray Conference, Plovidv, Bulgaria, 1977, International Cosmic Ray Conference, Vol. 11 (Bulgarian Academy of Sciences, Sofia, 1977), p. 132.

[2] A. R. Bell, Mon. Not. R. Astron. Soc. 182, 147 (1978).

[3] R. D. Blandford and J. P. Ostriker, Astrophys. J. Lett. 221, L29 (1978).

[4] P. Chen, T. Tajima, and Y. Takahashi, Phys. Rev. Lett. 89, 161101 (2002).

[5] Y. Lyubarsky, Astrophys. J. 652, 1297 (2006).

[6] M. Hoshino, Astrophys. J. 672, 940 (2008).

[7] Y. Kuramitsu and V. Krasnoselskikh, Phys. Rev. Lett. 94, 031102 (2005).

[8] H. Takabe, H. Nagatomo, A. Sunahara, N. Ohnishi, A. I. Mahdy, Y. Yoda, S. Naruo, H. Azechi, H. Nishimura, and K. Mima, Plasma Phys. Controlled Fusion 41, A75 (1999).

[9] B. A. Remington, D. Arnett, R. P. Drake, and H. Takabe, Science 284, 1488 (1999).

[10] R. P. Drake, J. Geophys. Res. 104, 14505 (1999).

[11] B. A. Remington, R. P. Drake, and D. D. Ryutov, Rev. Mod. Phys. 78, 755 (2006).

[12] T. Tajima and J. M. Dawson, Phys. Rev. Lett. 43, 267 (1979).

[13] A. Pukhov and J. Meyer-ter-Vehn, Appl. Phys. B: Lasers Opt. B 74, 355 (2002).

[14] S. P. D. Mangles et al., Nature (London) 431, 535 (2004).

[15] C. G. R. Geddes, C. Toth, J. van Tilborg, E. Esarey, C. B. Schroeder, D. Bruhwiler, C. Nieter, J. Cary, and W. P. Leemans, Nature (London) 431, 538 (2004).

[16] J. Faure, Y. Glinec, A. Pukhov, S. Kiselev, S. Gordienko, E. Lefebvre, J.-P. Rousseau, F. Burgy, and V. Malka, Nature (London) 431, 541 (2004).
[17] E. Miura, K. Koyama, S. Kato, N. Saito, M. Adachi, Y. Kawada, T. Nakamura, and M. Tanimoto, Appl. Phys. Lett. 86, 251501 (2005).

[18] A. Pukhov, Z.-M. Sheng, and J. Meyer-Ter-Vehn, Phys. Plasmas 6, 2847 (1999).

[19] C. Gahn, G. D. Tsakiris, A. Pukhov, J. Meyer-ter-Vehn, G. Pretzler, P. Thirolf, D. Habs, and K. J. Witte, Phys. Rev. Lett. 83, 4772 (1999).

[20] S. P. D. Mangles et al., Phys. Rev. Lett. 94, 245001 (2005).

[21] S. Kneip et al., Phys. Rev. Lett. 100, 105006 (2008).

[22] Y. Kuramitsu, Y. Sakawa, T. Kato, H. Takabe, and M. Hoshino, Astrophys. J. Lett. 682, L113 (2008).

[23] Y. Kitagawa et al., IEEE J. Quantum Electron. 40, 281 (2004).

[24] N. Nakanii et al., Appl. Phys. Lett. 93, 081501 (2008).

[25] K. A. Tanaka, T. Yabuuchi, T. Sato, R. Kodama, Y. Kitagawa, T. Takahashi, T. Ikeda, Y. Honda, and S. Okuda, Rev. Sci. Instrum. 76, 013507 (2005).

[26] N. Nakanii et al., Rev. Sci. Instrum. 79, 066102 (2008).

[27] T. Terasawa, Washington DC American Geophysical Union Geophysical Monograph Series, Vol. 61 (AGU, Washington DC, 1991), p. 277.

[28] Y. Kuramitsu and T. Hada, Geophys. Res. Lett. 27, 629 (2000).

[29] Y. Kuramitsu and V. Krasnoselskikh, J. Geophys. Res. [Space Phys.] 110, 10108 (2005).

[30] M. C. Begelman, R. D. Blandford, and M. J. Rees, Rev. Mod. Phys. 56, 255 (1984).

[31] N. H. Matlis et al., Nat. Phys. 2, 749 (2006). 